\title{
International Congress 'Hidden Hunger', March 5-9, 2013, Stuttgart-Hohenheim, Germany
}

\author{
Hans K. Biesalski \\ Department of Biological Chemistry and Nutrition, University Stuttgart-Hohenheim, Stuttgart, Germany
}

The international congress 'Hidden Hunger' brought more than 350 scientists and field workers (not-for-profit organizations, governmental and intergovernmental organizations, private business, and academic institutions) together to present and discuss 6 different topics related to hidden hunger: definition and assessment, magnitude and medical impact, worldwide reasons, the 1,000-day window, food-based programs, and solutions and future perspectives. There were 125 presentations including 25 keynote lectures, 80 posters, and 3 panel discussions. There was a strong emphasis on projects from Africa and Asia, which were presented in 45 oral presentations and 80 posters.

David Nabarro [coordinator of Scaling Up Nutrition (SUN) Movement] underlined that at the core of all efforts, women should be empowered to be leaders in their families and communities, leading the way to a healthier and stronger world. He also emphasized that specific actions for nutrition (feeding practices and behaviors, fortification of foods, micronutrient supplementation, and treatment of acute malnutrition) and nutrition-sensitive strategies (agriculture, clean water and sanitation, education and employment, and health care) need to be combined in a meaningful way.

\section{KARGER}

(c) 2013 S. Karger AG, Basel

0250-6807/13/0624-0298\$38.00/0

E-Mail karger@karger.com

www.karger.com/anm

\section{Definition and Assessment}

A greater understanding of how deficiencies manifest themselves and to what extent they are clinically relevant, by sex and age, remains important, and there is a priority need for improved standardization and reporting of measurements (Patrick Webb, Tufts University, Boston, Mass., USA).

The FAO (Food and Agriculture Organization of the United Nations) indicators of hidden hunger capture undernourishment but not inadequate intake of essential nutrients. The committee on world food security strongly recommends (October 2011) that the FAO improves its measure of undernourishment with special emphasis on improving the underlying data and parameters including the methodology (Eileen Kennedy, Tufts University, Boston, Mass., USA).

\section{Magnitude and Medical Impact}

Vitamin A, iron, iodine, and zinc deficiencies represent clear global priorities, based on their extent and benefits of prevention. Other essential micronutrient defi- 
ciencies with health consequences (e.g. vitamins D, E, K and B-complex, and selenium) almost certainly exist at varying levels of public health concern in undernourished societies. Yet, few have been widely assessed, and virtually none have gained prominent public health attention in developing countries, leaving evidence on how to prevent most micronutrient deficiencies grossly lacking (Keith West, Johns Hopkins University, Baltimore, Md., USA).

Hidden hunger and, in particular, vitamin A deficiency is a special issue for infants and children: the newborn has little vitamin A to conserve. Improving the nutrition of the mother may be very important for remedying vitamin A deficiency in young children, while also improving maternal health (Catharine Ross, Pennsylvania State University, University Park, Pa., USA).

According to Michael Zimmermann (ETH Zurich, Zurich, Switzerland), iodine deficiency or inadequate intake is a worldwide problem and affects nearly one third of the world's population. Despite worldwide fortification of salt with iodine, people in 31 countries are still iodine deficient.

\section{Worldwide Reasons}

According to Joachim von Braun (Center for Development Research, Bonn, Germany), the food price hikes in 2007-2008 and in 2011 were partly caused by rising consumer demand due to population and income growth, coupled with factors such as high oil prices, rise in demand for biofuel production, slow agricultural supply response, and malfunctioning financial systems and commodities markets. At the same time, poor consumers were negatively affected by the recent food price hikes and increased price volatility.

As in developing countries, hidden hunger is related to poverty in developed nations. Adam Drenowski (University of Washington, Seattle, Wash., USA) documented the role of food prices on food choices and resulting food insecurities. Hidden hunger in the United States and other developed countries is linked to profound inequalities in income and socioeconomic status. Food prices and diet costs mediate the observed links between poverty, obesity, and micronutrient deficiencies in the diet. First, hidden hunger and obesity are not mutually exclusive. Both nutrient deficiencies and obesity are most prevalent among population subgroups with low economic resources and high poverty rates. Second, the current hierarchy of food prices is such that energy-dense foods com-

International Congress 'Hidden Hunger' posed of refined grains, added sugars, or fats have become the least expensive sources of concentrated dietary energy. Not surprisingly, diets high in sugars and fats are cheaper than prudent diets based on lean meats, fish, fresh vegetables, and fruit. Lower-income and food-insecure persons have lower food expenditures, low fruit and vegetable consumption, and lower-quality diets. Third, the more nutrient-rich foods not only cost more (per MJ), but the price disparity between foods that are energy dense versus nutrient rich continues to grow. As a result, populations in developed countries are becoming undernourished yet overfed.

\section{1,000-Day Window}

Berthold Koletzko (Hauner Children's Hospital, University of Munich, Munich, Germany) emphasized that pre- and postnatal nutrition has a major impact on immediate outcomes (e.g. growth) and also a programming effect on long-term health, performance, and later disease risk up to old age.

Especially in the first year of life, maternal and child health and nutrition are strongly interconnected. Both nutritional and health aspects make breastfeeding the optimum choice. If babies are not breastfed, this increases the risk for malnutrition, infections, and other diseases. Breastfeeding needs protection by society, and all women who choose to breastfeed need to be supported (Eileen Rouw, Academy of Breastfeeding, Germany).

Maternal micronutrient status and/or intake in lactation have a major influence on the content of micronutrients in breast milk, especially that of vitamins A and $\mathrm{D}$, iodine, most $\mathrm{B}$ vitamins, and selenium. The public health importance of poor maternal micronutrient status in lactation has not been adequately studied or documented at this time, nor has the effectiveness of maternal supplementation to improve breast milk composition (Lindsay Allen, University of California, Davis, Calif., USA).

Addressing micronutrient deficiency in infants is a complex challenge. Whilst identified deficiencies require immediate supplementation, sustainable preventive interventions need careful consideration of the living conditions. The most promising preventive interventions seem to combine food security support and nutrition education (Michael Krawinkel, University Giessen, Giessen, Germany).

Data from research with food fortification using micronutrient powder (MNP) in Rwanda and Zambia pre- 
sented by Mélanie J. Suter (University of British Colombia, Vancouver, B.C., Canada) illustrates the importance of formative research and the role it must play to inform MNP interventions in different cultural contexts. Acceptability, utilization, and adherence are heavily dependent on culturally appropriate and specifically tailored communication materials and messaging. To optimize acceptability, utilization, and adherence of home fortification with MNP, formative research is essential to understand the context and design a culturally sensitive intervention and appropriate communication strategy.

\section{Food-Based Programs}

To accelerate progress in reducing micronutrient deficiencies, Marie Ruel [International Food Policy Research Institute (IFPRI), Washington, D.C., USA] called for leveraging multiple sectors, including health, agriculture, social protection and early child development, and focusing on empowering women. The ultimate benefit of diversifying the platforms used to deliver micronutrient interventions is that it will allow tackling micronutrient deficiencies while simultaneously addressing several of their underlying drivers such as poverty, food insecurity, gender inequality, and lack of access to basic services.

Nutrition-sensitive agriculture and food-based approaches are advocated that increase availability of, access to, and consumption of an adequate quantity of food in terms of calories and in quality in terms of variety, diversity, nutrient content, and food safety. Such approaches are considered to be sustainable ways of improving the nutritional status of poor, marginalized, socially excluded and vulnerable populations. It is of great importance to close the 'nutrition gap', i.e. the gap between what foods are currently available and what foods ought to be available in terms of macro- and micronutrient content to achieve improved diets and thereby improved nutrition, as highlighted by Brian Thompson (FAO, Rome, Italy).

Nutritionists, while usually focused on concerns of immediate importance, are increasingly challenged to ensure the long-term viability of healthy diets, an important message from Timothy Johns (McGill University, Montreal, Que., Canada). Principles of sustainability that define agendas for research and action call on nutrition to engage in new multidisciplinary partnerships. Sustainable diets in ensuring food security and the availability of micronutrients and health-mediating constituents can provide the foundation for healthy diets and lifestyles both in the present and future. Within transdisciplinary approaches, nutritionists are recognized as revealing perspectives essential for global transformation that refines local and global links between production and consumption, and the interests of people of rich and poor countries alike.

Eliminating hidden hunger is intrinsically valuable based on three core ideas: (1) many of the solutions to hidden hunger have instrumental value, that is, they are investments that yield a high rate of economic return, but (2) their instrumental value is not a substitute for the intrinsic rationale, and (3) economic estimates of these returns are subject to considerable uncertainty (John Hoddinott, IFPRI, Washington, D.C., USA).

Poverty, hunger, and gross food insecurity are ravaging the masses not because of lack of resources in some cases, but due to the absence of a 'messiah' or a true patriot or selfless advisors and managers of national resources and tax payers' money (Florence N. Uchendu, Department of Health Sciences and Nutrition, National Open University of Nigeria, Lagos, Nigeria).

A sustainable solution to the food price crisis needs a holistic approach encompassing the whole agri-food system. Interventions intended to mitigate adverse effects on nutrition of poor consumers should be coupled with supply side interventions intended to sustainably increase food production and market policies preventing excessive price volatility, thereby serving poor consumers.

\section{Solutions and Future Perspectives}

Many groups have argued that the response of food producers to the challenge of food security should take the form of 'sustainable intensification'. However, critics have argued that sustainable intensification is just business-as-usual or internally contradictory, or ignores other major food system agendas such as ending hidden hunger. Sustainable intensification, if interpreted appropriately, is a radical approach to food production and meshes well with goals such as improving nutrition (Charles Godfray, University of Oxford, Oxford, UK).

Fully utilizing nutrient-rich NTCs (national and traditional crops) to combat hidden hunger will require locally focused, interdisciplinary, and problem-oriented approaches. Further, these circumstances imply the need to recognize the role of rural-development nongovernmental organizations (NGOs) not just in educating nu- 
trient-poor populations, but also in driving cultural change (Alder Keleman, Yale University, New Haven, Conn., USA).

\section{NGO Forum}

A HarvestPlus panel discussion concluded that nutrition research of crops that have been traditionally bred to contain more micronutrients has amassed an important body of scientific evidence to support the construct that biofortification is an efficient and effective strategy to alleviate hidden hunger and should be adopted soon as another mainstream intervention to prevent vitamin and mineral deficiencies.

The potential of food fortification to reduce the nutrient intake gap during the 1,000-day window of opportunity (from conception to a child's second birthday) as well as throughout the whole life cycle was debated by GAIN (Global Alliance for Improved Nutrition). It is important to prepare women of reproductive age, in particular adolescent girls, for future pregnancies and to ensure that children receive enough nutrients (micro and macro) for an optimal start of their lives. Exclusive breastfeeding is recommended until about 6 months of age, followed by adequate complementary foods. Implemented appropriately, large-scale food fortification has the potential to contribute significantly to the nutrient intake of children from 6 months onwards.

A Sight and Life symposium addressed folate and vitamin D inadequacy. Both folate and folic acid are essential for maintaining optimal health, but folic acid is significantly more stable and bioavailable than folate. Perhaps the most well-known benefit of folic acid is its ability to reduce the risk of neural tube defects. Given that many populations have low folate intakes and women of childbearing age often fail to take folic acid during the critical periconceptional period, flour fortification with folic acid has been implemented by about 60 countries around the world. The ability of folic acid to reduce neural tube defects is well documented and accepted. A concern regarding cancer risk is often attributed to an ecological study which claimed a temporal association between the start of mandatory flour fortification in the United States and Canada and an increase in colorectal cancer. However, findings from a recent meta-analysis show that folic acid, even when consumed at doses above the upper intake level over a sustained period of time, is not associated with an increased cancer risk.

\section{Agri-Business Forum}

BASF

A prominent example of such partnership work is SAFO, the Strategic Alliance for the Fortification of Oils and Staple Foods, driven by GIZ (Gesellschaft für Internationale Zusammenarbeit) and BASF with a cluster of smaller partners. SAFO already reached its objective to improve access to vitamin A-fortified staple food for more than 150 million people at risk of malnutrition. With the German Food Partnership, a broad BMZ (Bundesministerium für wirtschaftliche Zusammenarbeit)and Gates Foundation-funded approach about to be kicked off, companies try to improve the whole agro-nutrition value chain in developing countries in a multipolar public-private partnership.

\section{DSM}

Compelling evidence shows that improving nutrition protects health, prevents disability, boosts economic productivity, and saves lives. Investments to support and improve the nutritional wellbeing of the most at-risk population groups, including children, women in child-bearing age, women and the elderly, yield benefits in the near term as well as greater long-term rewards.

\section{Nestlé}

A total of 13 studies reported outcomes related to iron fortification in women and revealed significant benefits on hemoglobin [standard mean difference (SMD) 0.64, 95\% CI 0.30, 0.97], serum ferritin concentrations (SMD $0.41,95 \%$ CI $0.23,0.60$ ), and on prevalence of anemia (risk ratio $0.68,95 \%$ CI $0.49,0.93$ ). Our review confirms the effectiveness of iron fortification strategies. The decision to use one strategy versus another would be based on programmatic opportunities, feasibility, and cost effectiveness. Iron, vitamin A, and zinc deficiencies lead to substantial costs in terms of direct medical costs (29 million USD), production losses (of 462 million USD), and disability-adjusted life years (116,656 DALYs) in 6- to 59-month-old children in the Philippines. Costs are highly concentrated in the lower socioeconomic strata and in children 6- to 23-months old. Multimicronutrient-fortified milk and cereal products can be an effective option to reduce anemia of children up to 3 years of age as risk of anemia is reduced by $57 \%$ (absolute risk reduction $22 \%$; number needed to treat 5 ). 


\section{Future Perspectives and Possible Solutions}

The final discussion with field workers and program leaders underlined the importance of a holistic approach to combat hidden hunger. Solution-oriented research including aspects of human behavior is needed along with a broad variety of concepts. Research approaches should take differences between countries into account and consider regional and local needs, as well as working with the population. All studies should be registered in clinical trial databases. Better communication is needed between scientists, policy makers, NGOs, and local population. Proactive strategies should bring different actors together. Gaps in coordination and communication should be identified.

There is a need for indicators of diet quality, harmonization of nutrient intake recommendations, and critical ethical reviews on different approaches, e.g. supplemen- tation, sprinkles, and fortification. Nutrition intervention and supplementation should be linked to and harmonized with other approaches such as vaccination, prevention and treatment of viral, bacterial and parasitic infections, and others. The farming environment and toxic factors such as mycotoxins need to be addressed.

The economic aspects of nutrition need to be considered. Stakeholders and planners from different backgrounds should collaborate, e.g. using sectorial approaches to coordinate different actors or by interministerial coordination committees.

A need was identified to share the results and challenges discussed at the conference with the broader public. To this end and to allow the sharing of study protocols and data, an electronic platform is being established that will be accessible through www.hiddenhunger.uni-hohenheim.de. 